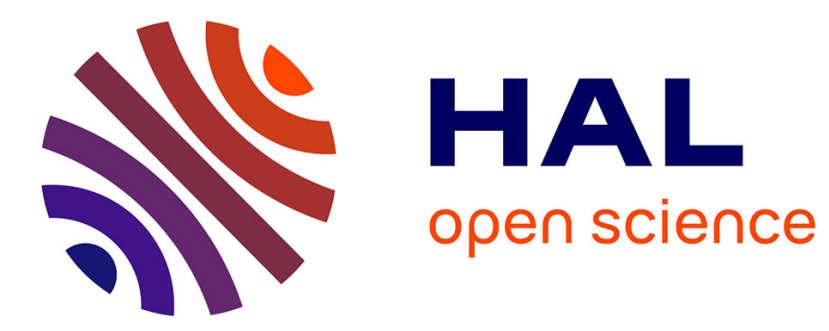

\title{
Complete Set of Invariants of a 4th Order Tensor: The 12 Tasks of HARDI from Ternary Quartics
}

\author{
Théodore Papadopoulo, Aurobrata Ghosh, Rachid Deriche
}

\section{To cite this version:}

Théodore Papadopoulo, Aurobrata Ghosh, Rachid Deriche. Complete Set of Invariants of a 4th Order Tensor: The 12 Tasks of HARDI from Ternary Quartics. Medical Image Computing and Computer-Assisted Intervention - MICCAI 2014, Sep 2014, Boston, United States. pp.233 - 240, 10.1007/978-3-319-10443-0_30. hal-01092492

\section{HAL Id: hal-01092492 https://hal.science/hal-01092492}

Submitted on 8 Dec 2014

HAL is a multi-disciplinary open access archive for the deposit and dissemination of scientific research documents, whether they are published or not. The documents may come from teaching and research institutions in France or abroad, or from public or private research centers.
L'archive ouverte pluridisciplinaire HAL, est destinée au dépôt et à la diffusion de documents scientifiques de niveau recherche, publiés ou non, émanant des établissements d'enseignement et de recherche français ou étrangers, des laboratoires publics ou privés. 


\title{
Complete Set of Invariants of a $4^{\text {th }}$ Order Tensor: The 12 Tasks of HARDI from Ternary Quartics
}

\author{
Théo Papadopoulo, Aurobrata Ghosh, and Rachid Deriche \\ Athena Project Team, Inria Sophia Antipolis - Méditerranée, France*
}

\begin{abstract}
Invariants play a crucial role in Diffusion MRI. In DTI $\left(2^{\text {nd }}\right.$ order tensors), invariant scalars (FA, MD) have been successfully used in clinical applications. But DTI has limitations and HARDI models (e.g. $4^{\text {th }}$ order tensors) have been proposed instead. These, however, lack invariant features and computing them systematically is challenging.

We present a simple and systematic method to compute a functionally complete set of invariants of a non-negative 3D $4^{\text {th }}$ order tensor with respect to $\mathrm{SO}_{3}$. Intuitively, this transforms the tensor's non-unique ternary quartic (TQ) decomposition (from Hilbert's theorem) to a unique canonical representation independent of orientation - the invariants.

The method consists of two steps. In the first, we reduce the 18 degrees-of-freedom (DOF) of a TQ representation by 3 -DOFs via an orthogonal transformation. This transformation is designed to enhance a rotation-invariant property of choice of the $3 \mathrm{D} 4^{t h}$ order tensor. In the second, we further reduce 3 -DOFs via a $3 \mathrm{D}$ rotation transformation of coordinates to arrive at a canonical set of invariants to $\mathrm{SO}_{3}$ of the tensor.

The resulting invariants are, by construction, (i) functionally complete, (ii) functionally irreducible (if desired), (iii) computationally efficient and (iv) reversible (mappable to the TQ coefficients or shape); which is the novelty of our contribution in comparison to prior work.

Results from synthetic and real data experiments validate the method and indicate its importance.
\end{abstract}

Keywords: Invariants, $\mathrm{SO}_{3}, 4^{\text {th }}$ order tensors, ternary quartics, orthogonal \& rotation transforms, canonical representation.

\section{Introduction}

High angular resolution diffusion imaging (HARDI) has vastly improved our analysis of the brain's microstructure and the detection of crossing-fibers from diffusion MRI (dMRI) where classical diffusion tensor imaging (DTI) is limited. Nonetheless, to assess the integrity of the white-matter affected by development, aging or neuro-degenerative pathologies, it is crucial to compute rotation invariant scalars or biomarkers. Although numerous invariant scalars are known for the $2^{\text {nd }}$ order DTI tensor $\mathbf{D}$, e.g. FA, MD, etc. 1] and have been successfully

\footnotetext{
* This work has been partially supported by the ANR project Mosifah (MN Program).

P. Golland et al. (Eds.): MICCAI 2014, Part III, LNCS 8675, pp. 233240 2014.

(c) Springer International Publishing Switzerland 2014
} 
used in clinical applications, few invariant scalars are available for HARDI. Although richer in description, higher order HARDI models lack comprehensive invariant scalar descriptors to decipher that information. Therefore, computing HARDI invariants is an important problem.

HARDI models are often estimated in the spherical harmonic (SH) or the bijective higher order tensor (HOT) bases. (HOTs are homogeneous polynomials and their SH transform provides a bijective map to SHs). Thus, studying invariant features of the SH or HOT bases has wide applications. Early proposals of HARDI biomarkers based on these bases can be found in [2, 3]. Though these are popular, they only recover a few of the possible invariant scalar biomarkers.

In the case of the $4^{\text {th }}$ order tensor (HOT4), equivalently SHs, more systematic approaches are presented in [4-6] but only six of the twelve invariants are found. [4] also presents D-eigenvalues which are the extrema of the HOT4. Further, [6] introduces the integrity basis and the idea of polynomial completeness.

From this, a more general idea presents itself - functional completeness: "find a set of invariants such that all other invariants are functions of the invariants of the set." Naturally, this leads to the idea of functional irreducibility: "what is the smallest such set?" How to find the minimal set of invariants required to completely describe the shape of a HOT4? The importance of this question can be illustrated on the DTI tensor. A functionally complete \& irreducible invariant set of $\mathbf{D}$ is its eigenvalue-set $\left\{\lambda_{1}, \lambda_{2}, \lambda_{3}\right\}$. However, if only $\left\{\lambda_{1}, \lambda_{2}\right\}$ are found and any number of invariant functions of $\left\{\lambda_{1}, \lambda_{2}\right\}$ (even greater than 3 ), it is impossible to describe the shape of $\mathbf{D}$ without $\lambda_{3}$. And there exist infinitely many such invariant functions!

Further noteworthy results for HOT4 are proposed in 7], where the cardinality of the irreducible set is shown to be 12 . The paper presents polynomial invariants and tries to establish polynomial completeness. However, this is only partially successful and the polynomial formulae are intractable and cannot be inverted to recompute the HOT4 coefficients, hence the approach is irreversible. [8] is based on rank-4 SHs and proposes a set of 25 invariants (and ad-hoc functions of these). However, no proof of functional completeness, irreducibility or reversibility is attempted (since only 12 invariants are required).

In this paper, we consider non-negative HOT4s and their Ternary Quartic (TQ) parameterization from Hilbert's theorem [9, 10] since the physical constraint of positivity arises commonly in dMRI, e.g. the diffusion kurtosis tensor (DKT) [11], the apparent diffusion coefficient tensor (ADC), or the Cartesian tensor fiber orientation distribution (CT-FOD).

We propose a simple and systematic method to compute a functionally complete set of invariants to $\mathrm{SO}_{3}$ of a non-negative HOT4 by mapping its non-unique TQ decomposition to a unique canonical representation independent of orientation. From this we extract the invariants. This involves a two step reduction process via an orthogonal and a rotation transform. The resulting invariants are, by construction, (i) functionally complete, (ii) functionally irreducible (if desired), (iii) efficient to compute and (iv) reversible. Reversibility ensures that the shape of the TQ can be inferred from the invariants and all prior/other 
invariants can be expressed in terms of these new ones. We validate the method on synthetic and real data tests to highlight its importance.

\section{Materials and Methods}

Background. Positivity is a common constraint in dMRI. The right way to parameterize a non-negative $3 \mathrm{D} 4^{\text {th }}$ order tensor $\mathcal{A}$, is a sum-of-squares as shown by Hilbert's theorem on non-negative TQ's $[9,10]$ :

$$
F_{4}(\mathbf{x})=\sum_{i+j+k=4} \mathcal{A}_{i, j, k} x^{i} y^{j} z^{k}=\left(\mathbf{v}^{T} \mathbf{w}_{1}\right)^{2}+\left(\mathbf{v}^{T} \mathbf{w}_{2}\right)^{2}+\left(\mathbf{v}^{T} \mathbf{w}_{3}\right)^{2}=\mathbf{v}^{T} \mathbf{W} \mathbf{W}^{T} \mathbf{v},
$$

where $\mathbf{x}^{T}=[x, y, z], \mathbf{v}^{T}=\left[x^{2}, y^{2}, z^{2}, \sqrt{2} x y, \sqrt{2} x z, \sqrt{2} y z\right], \mathbf{w}_{i}(i=1 . .3)$ are $6 \times 1$ vectors of the coefficients of the three quadratic forms and $\mathbf{W}=\left[\mathbf{w}_{\mathbf{1}}\left|\mathbf{w}_{\mathbf{2}}\right| \mathbf{w}_{\mathbf{3}}\right]$ is a $6 \times 3$ matrix. The TQ parameterization has $18=6 \times 3$ independent coefficients, although $\mathcal{A}$ has only 15 . These 3 degrees-of-freedom (DOFs) imply that $\mathbf{W}$ and $\mathbf{W S}$ for any $3 \times 3$ orthogonal matrix $\mathbf{S}(3-\mathrm{DOFs})$ result in the same $F_{4}$. To resolve this ambiguity, [9] proposed a QR/Iwasawa decomposition of $\mathbf{A}$, the top $3 \times 3$ block of $\mathbf{W}$, which fixes $\mathbf{S}$ such that $\mathbf{A}$ becomes triangular - effectively zeroing out 3 coefficients. Here, we introduce a slightly different notation:

$$
F_{4}(\mathbf{x})=\sum\left(\mathbf{x}^{T} \mathbf{C}_{i} \mathbf{x}\right)^{2}=\|\mathbf{c}\|^{2}, \quad i=1 . .3,
$$

where $\mathbf{C}_{i}$ are $3 \times 3$ symmetric matrix representations of vectors $\mathbf{w}_{i}$ and $\mathbf{c}^{T}=$ $\left[\mathbf{x}^{T} \mathbf{C}_{1} \mathbf{x}, \mathbf{x}^{T} \mathbf{C}_{2} \mathbf{x}, \mathbf{x}^{T} \mathbf{C}_{3} \mathbf{x}\right]$. In this notation $F_{4}(\mathbf{x})=\mathbf{c}^{T} \mathbf{c}=\widehat{\mathbf{c}}^{T} \widehat{\mathbf{c}}$, where $\widehat{\mathbf{c}}=\mathbf{S c}$.

Theory. TQ's have 18-DOFs, while HOT4s have 15-DOFs. The excess 3-DOFs can be eliminated by fixing an orthogonal transformation $\mathbf{S}$. Additionally a rotation invariance criterion would further eliminate 3-DOFs via a rotation transformation $\mathbf{R}$, of coordinates, resulting in the known 12-DOFs.

The basic idea is, therefore, a two step reduction process to twice remove 3-DOFs - first an orthogonal transform $\mathbf{S}$ and second a rotation transform $\mathbf{R}$ - resulting in a canonical representation of a TQ invariant to rotations: $\underbrace{\mathbf{C}_{18 \text {-DOF }} \rightsquigarrow \mathbf{S}_{3 \text {-DOF }}}_{\text {Step-1 }} \Longrightarrow \underbrace{\mathbf{C}_{15-\mathrm{DOF}} \rightsquigarrow \mathbf{R}_{3 \text {-DOF }}}_{\text {Step-2 }} \Longrightarrow \mathbf{C}^{\prime \prime}{ }_{12 \text {-DOF }} \longmapsto 15$ invariants (complete set) $\rightarrow 12$ invariants (complete \& irreducible set if desired).

Orthogonal Transform (S). Contrary to [9], we do not choose $\mathbf{S}$ to zero out 3 coefficients, since that is not invariant to $\mathrm{SO}_{3}$ and the zeros would be lost after the second rotation transform step. Instead, we design $\mathbf{S}$ to enhance a property of choice that is rotation-invariant. First we provide an overview of the approach before describing the rotation-invariant properties.

The application of $\mathbf{S}$ on $\mathbf{c}$ results in the transformations $\mathbf{C}_{i}{ }_{i}=\sum_{j} S_{i j} \mathbf{C}_{j}, i, j=$ 1..3. Hence, each $\mathbf{C}_{i}^{\prime}\left(\sigma_{i}\right)$ is a function of the $i^{\text {th }}$ row vector $\sigma_{i}=\left[S_{i 1}, S_{i 2}, S_{i 3}\right]$ of $\mathbf{S}$. If $\mathbf{S}$ is required to enhance a chosen property $\mathcal{P}$ of $\left\{\mathbf{C}^{\prime}{ }_{i}\right\}_{i=1}^{3}$ and if $\mathcal{P}$ is a quadratic function, then $\mathcal{P}$ can be written as: $\mathcal{P}\left(\left\{\mathbf{C}^{\prime}{ }_{i}\left(\sigma_{i}\right)\right\}\right)=\frac{1}{2} \sigma \mathbf{K} \sigma^{T}$, 
where $\mathbf{K}$ is the Hessian of the quadratic function $\mathcal{P}$. To enhance $\mathcal{P}$ it suffices to find its extrema or to diagonalize $\mathbf{K}=\mathbf{U} \Lambda \mathbf{U}^{T}$. $\mathbf{K}$ being symmetric, $\mathbf{U}$ is an orthogonal matrix whose columns either maximize or minimize $\mathcal{P}$ depending on the order of the corresponding eigenvalues in $\Lambda$. Hence, if $\mathbf{S}$ is chosen to be $\mathbf{U}^{T}$, $\mathbf{S}$ would enhance $\mathcal{P}$ when computing $\left\{\mathbf{C}_{i}^{\prime}\right\}$ from $\left\{\mathbf{C}_{i}\right\}$. In summary, to enhance a quadratic property via an orthogonal transform $\mathbf{S}$, we simply need to choose $\mathbf{S}$ as the eigenvector matrix $\mathbf{U}^{T}$ from the eigen-decomposition of $\mathbf{K}$.

Let us now examine rotation invariant properties of $\left\{\mathbf{C}_{i}^{\prime}\right\}$. Since, $\left\{\mathbf{C}_{i}^{\prime}\right\}$ are all $3 \times 3$ symmetric matrices, their rotation-invariant properties are simply the coefficients of their characteristic polynomials: $M_{1}\left(\mathbf{C}^{\prime}{ }_{i}\left(\sigma_{i}\right)\right)=\operatorname{trace}\left(\mathbf{C}^{\prime}{ }_{i}\left(\sigma_{i}\right)\right)$, $M_{2}\left(\mathbf{C}_{i}^{\prime}\left(\sigma_{i}\right)\right)=\sum_{k_{1}, k_{2}} \mu_{k_{1}} \mu_{k_{2}}$, where $\mu_{k}$ are the eigenvalues of $\mathbf{C}_{i}^{\prime}\left(\sigma_{i}\right)$, and $M_{3}\left(\mathbf{C}_{i}^{\prime}\left(\sigma_{i}\right)\right)=\operatorname{det}\left(\mathbf{C}^{\prime}{ }_{i}\left(\sigma_{i}\right)\right)$. $M_{1}$ is a linear function of $\sigma_{i}, M_{2}$ is a quadratic function, while $M_{3}$ a cubic function. Hence $M_{1}^{2} \& M_{2}$ are two quadratic functionproperties (i.e. $\mathcal{P}$ ) that are also rotation-invariant.

Let us name $\mathbf{H}_{1}$ and $\mathbf{H}_{2}$ the two Hessian matrices of $M_{1}^{2}$ and $M_{2}$ respectively. To compute the corresponding orthogonal transforms $\mathbf{S}$ that enhance these properties we need to calculate $\mathbf{H}_{1}$ and $\mathbf{H}_{2} . \mathbf{H}_{1}$ is simply $\mathbf{H}_{1}=2 \mathbf{T} \mathbf{T}^{T}$, where $\mathbf{T}^{T}=\left[\operatorname{trace}\left(\mathbf{C}^{\prime}{ }_{1}\right)\right.$, trace $\left(\mathbf{C}^{\prime}{ }_{2}\right)$, trace $\left.\left(\mathbf{C}^{\prime}{ }_{3}\right)\right]$. The form of $\mathbf{H}_{2}$ is more involved but is simple to derive on a computer-algebra-system and can be provided on request.

Interestingly, any combination of $M_{1}$ and $M_{2}$ that is a quadratic function is also a rotation invariant property. Linear combinations of $M_{1}^{2}$ and $M_{2}$ are simple to compute as linear combinations of $\mathbf{H}_{1}$ and $\mathbf{H}_{2}$. In particular, we consider the following invariant properties:

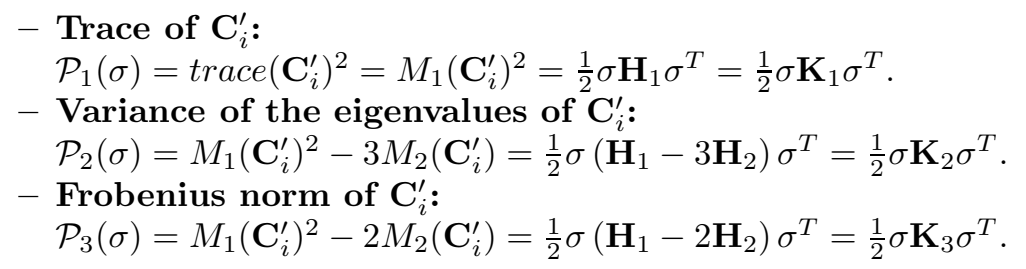

Thus, it is possible to enhance any of these properties while designing an orthogonal transform $\mathbf{S}$ in the first reduction step. However, this is a generic design and many other properties could be considered. $\mathcal{P}_{2}$ in particular is the same as the numerator of FA for DTI. However, in this case $\left\{\mathbf{C}_{i}^{\prime}\right\}$ may have negative eigenvalues since their squares are considered in the TQ representation. Here, we only present results of $\mathbf{K}_{2}$ though we have experimented with all three properties.

It is important to note that in choosing $\mathbf{S}$ to enhance a rotation-invariant property, contrary to [9], the application of $\mathbf{S}$ to the TQ will not result in 3 coefficients becoming null. It will reduce 3-DOFs of the TQ but will still result in 18 non-null coefficients, where 3 are now dependent.

Rotation Transform $(\mathbf{R})$. The second reduction step involves further eliminating 3-DOFs via a rotation transformation of the coordinates to map the modified TQ coefficients $\left\{\mathbf{C}_{i}^{\prime}\right\}$ to a representation that is independent of orientation. We choose this rotation transform $\mathbf{R}$ from the eigen-decomposition of $\mathbf{C}_{1}^{\prime}=\mathbf{R} \Sigma \mathbf{R}^{T}$. 
$\mathbf{C}_{1}^{\prime}$ is diagonalized and the other two $\mathbf{C}_{i}^{\prime}$ 's are transformed as $\mathbf{C}_{i}^{\prime \prime}=\mathbf{R}^{T} \mathbf{C}_{i}^{\prime} \mathbf{R}$. The two transformations, $\mathbf{S}$ and $\mathbf{R}$ combined, map the original TQ parameterization to a canonical representation that is invariant to all $3 \mathrm{D}$ rotations.

This results in 15 non-null coefficients $\left(\mathbf{C}_{1}^{\prime \prime}=\Sigma: 3, \mathbf{C}_{2 \& 3}^{\prime \prime}: 2 \times 6\right)$ with $12-$ DOFs, implying that three are dependent. This, however, ensures functional completeness and, by construction, the invariants are also reversible and still maintain the TQ structure.

The process for extracting the irreducible set of 12 invariants involves considering the off-diagonal terms of the matrix $\mathbf{K}$. This yields a linear system in the coefficients of $\mathbf{C}_{i}^{\prime \prime}$, which can be solved to express 3 coefficients as an expression of the others. However, the choice of which 3 coefficients to eliminate is not unique. This ambiguity implies that the space of TQs is covered by several maps and that there are many ways to extract the irreducible set of 12 invariants from the 15 non-null (invariant) coefficients. Therefore, although for theoretical purposes it is possible to extract the irreducible set of 12 invariants, in practice it is more convenient to work directly with the 15 invariant coefficients with slightly redundant information but guaranteed functional completeness.

Unicity of Sign. The two steps involving $\mathbf{S}$ and $\mathbf{R}$ proceed via eigen-decompositions of $\mathbf{K}$ and $\mathbf{C}_{1}^{\prime}$, implying that the eigenvectors in $\mathbf{U}$ and $\mathbf{R}$ are unique only up to a sign. This introduces an ambiguity since a large set of maps can be used to transform the TQ coefficients to the canonical representation. These arbitrary sign changes can become difficult to handle in the presence of noise or when values are close to zero. We devised the following procedure to minimize this ambiguity. The sign of the columns of $\mathbf{U}$ are flipped to ensure that the largest absolute eigenvalue of the corresponding $\mathbf{C}_{i}^{\prime}$ is positive. The sign of the columns of $\mathbf{R}$ are flipped to ensure that the largest absolute entry in the column is positive. However, since $\mathbf{R}$ is a rotation matrix, if the above operation results in $\operatorname{det}(\mathbf{R})<0$, the sign of $\mathbf{R}$ is flipped to guarantee a proper rotation.

So far in this section, we presented the coefficients of the quadratic forms (TQ coefficients) modified by the orthogonal and rotation transforms as the invariants. However, any functions of these invariant coefficients are also valid invariants. In practice, on real data, we found that working with "super-features" such as $M_{1}, M_{2}, M_{3}$ of the final canonical coefficients to be more robust to rotation tests. Note that these still satisfy functional completeness but reversibility becomes harder to establish. Nonetheless, it is important to explore suitable "super-feature" functions, especially from a physical/physiological perspective.

\section{$3 \quad$ Experiments and Results}

We conducted tests on synthetic and real data to verify the invariance of the canonical invariants to rotation. In the synthetic data experiments, we used the multi-tensor model to generate voxels with various crossing configurations $(1,2$ $\& 3)$. These were then arbitrarily rotated in space before estimating the HOT4s and the invariants. We conducted a similar real data experiment from an in vivo acquisition where we rotated the set of gradient directions arbitrarily before 

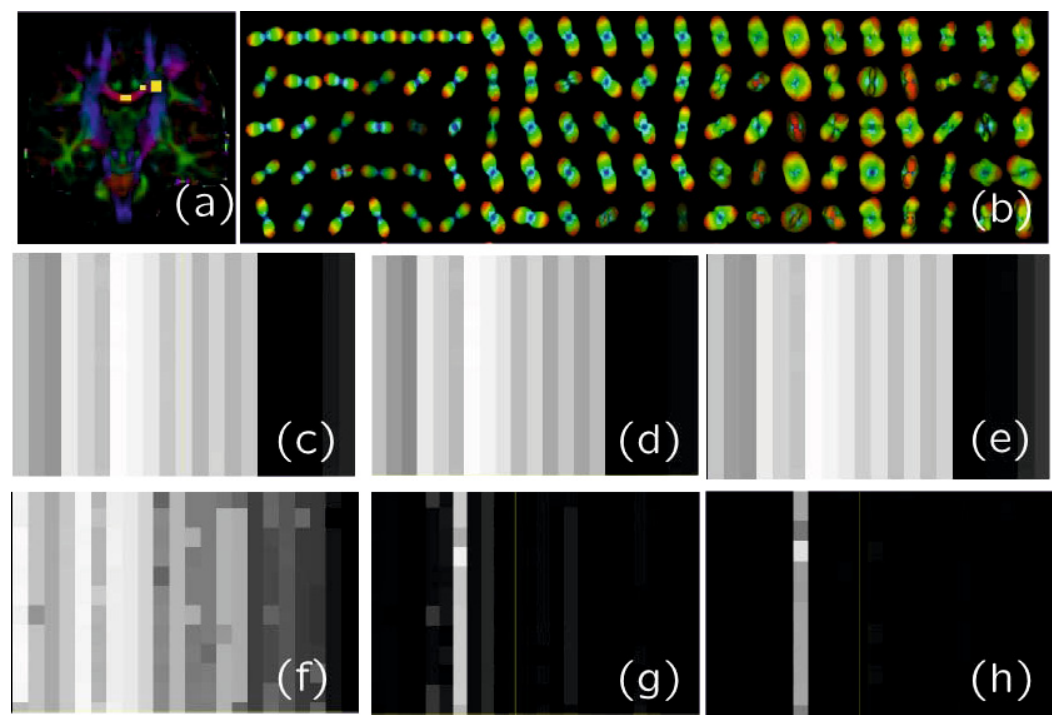

Fig. 1. Real data (qualitative): Rotation invariance test and comparison with principal invariants (PIs). (a) Coronal slice indicating chosen voxels. (b) 1st row: the sample voxels, followed by samples with random rotations. (c-e): 3 PIs. (f-h) 3 new/canonical invariants. PIs (c-e) resemble each other or capture similar information. The new invariants (f-h) have a richer spectrum and as expected capture more information.

estimating the HOT4s and the invariants. In both cases, the results were very similar. Hence, we present only the real data results here.

The real data was acquired with a whole-body 3 Tesla Magnetom TRIO scanner (Siemens Medical Solution). It was equipped with an 8-channel head array coil. The twice-refocused spin-echo EPI sequence $(\mathrm{TR}=12 \mathrm{~s}, \mathrm{TE}=100$ ms, $128 \times 128$ image matrix, FOV $=22.0=22.0 \mathrm{~cm}^{2}$ ) consisted of 60 diffusionencoding gradient directions with a $b$-value of $1000 \mathrm{~s} / \mathrm{mm}^{2}$ [12].

In the experiment, we chose 21 voxels from a coronal slice with some voxels in the Corpus Callosum (CC) and others in a region where radial projections of the CC intersect the superior longitudinal fasciculus (SLF) and the corticospinal tract (CST), Fig. 17. This allowed us to consider voxels with $1,2 \& 3$ fiber crossings. For each of these voxels, we randomly rotated the acquisition gradients 50 times to generate 50 test cases. From these, we estimated the nonnegative HOT4s or TQs, Fig. [o, and computed the principal invariants [6] and the new/canonical invariants. Some of these are in Figs. 11k-h. Finally, we computed the difference between each pair of 50 test cases for all the voxels to compute the average relative error for both the principal \& new invariants. This quantitative result is presented in Fig. 2 .

In Figs. 11-h we notice that the principal invariants (mid-row) capture almost the same information, while the new invariants (bot-row) have a richer spectrum. The new invariants look more noisy but this is only due to the visual 


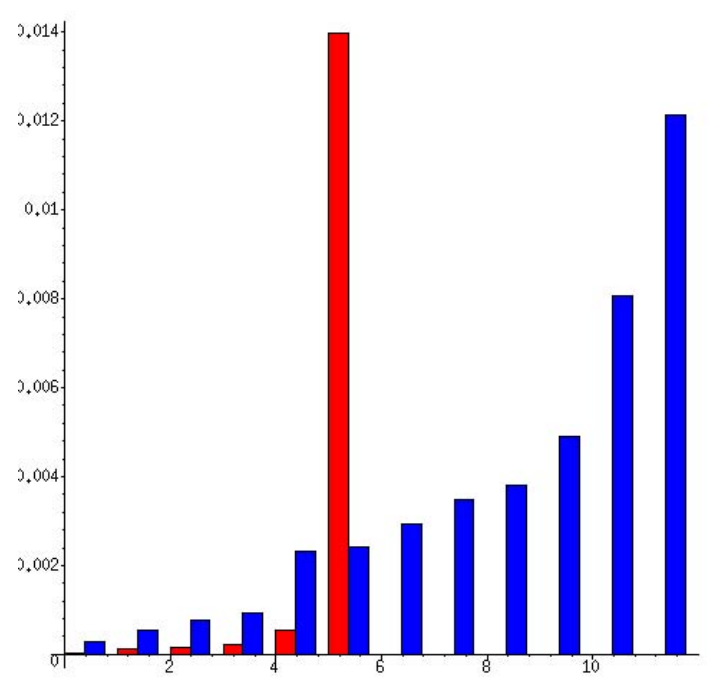

Fig. 2. Real data (quantitative): Rotation invariance test and comparison with principal invariants (PIs). Average relative errors between the new/canonical invariants compared to PIs (see Fig. 11). Blue-bars: new/canonical invariants (only a few are shown). Red-bars: principal invariants. All invariants (new \& PIs) are stable under rotation and commit less than $2 \%$ error even in the worst case.

representation. From Fig. 2 it is clear that all the (new \& principal) invariants are stable under rotation and commit less than $2 \%$ error on an average even in the worst case. Thus, our tests reveal that the new invariants are stable and as expected capture more invariant/shape information than principal invariants.

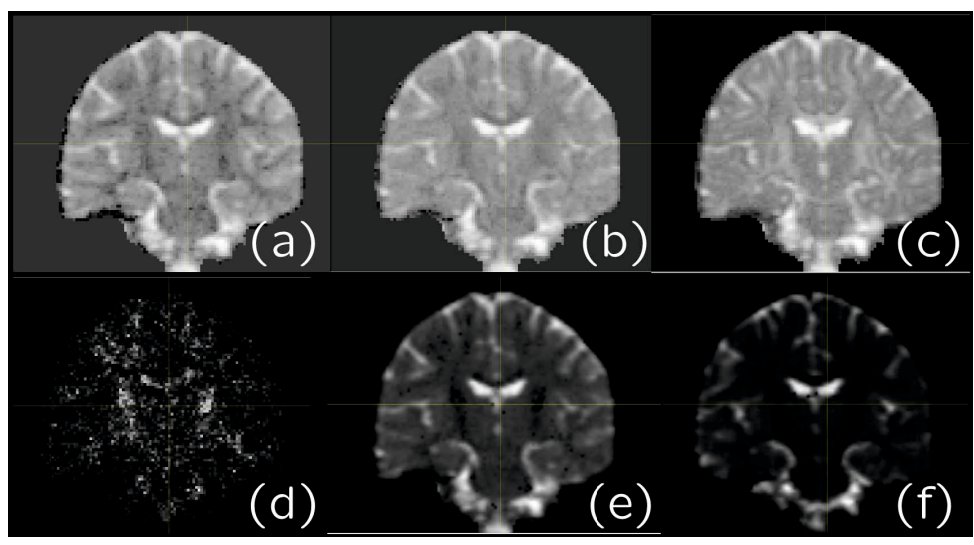

Fig. 3. A sample of the new/canonical invariants. Six are displayed on a coronal slice. 


\section{Conclusion}

We have proposed a simple and systematic method for computing a functionally complete set of invariants of a non-negative HOT4. The method has two steps. First, we designed an orthogonal transform to enhance rotation-invariant properties of the tensor while eliminating 3-DOFs from its TQ representation. Second, we chose a rotation transform to map the TQ coefficients to a canonical representation invariant to orientation. From this, we extracted the invariants.

These canonical invariants are, by construction, (i) functionally complete, (ii) functionally irreducible (if desired) - although in practice we deal with a slightly redundant super-set, (iii) efficient to compute and (iv) reversible - allowing us to map the TQ's shape completely. Reversibility ensures that all other/prior invariants can be described as functions of these canonical invariants.

We conducted tests on synthetic and real data and validated the invariants. The results revealed that the new invariants were stable under rotation and captured a rich spectrum of information. In the future, we plan to explore physiological interpretations and to conduct a more elaborate clinical validation.

\section{References}

1. Basser, P.J.: Inferring microstructural features and the physiological state of tissues from diffusion-weighted images. NMR in Biomedicine 8, 333-344 (1995)

2. Özarslan, E., Vemuri, B.C., Mareci, T.H.: Generalized scalar measures for diffusion MRI using trace, variance and entropy. Mag. Res. in Med. 53(4), 866-876 (2005)

3. Tuch, D.: Q-ball imaging. Mag. Res. in Med. 52(6), 1358-1372 (2004)

4. Qi, L., Han, D., Wu, E.: Principal invariants and inherent parameters of diffusion kurtosis tensors. J. of Math.l Anal. and Ap. 349(1), 165-180 (2009)

5. Fuster, A., van de Sande, J., Astola, L., Poupon, C., Velterop, J., Romeny, B.M.t.H.: Fourth-order Tensor Invariants in High Angular Resolution Diffusion Imaging. In: Zhang, G., Adluru, N (eds.) CDMRI Workshop, MICCAI. LNCS, vol. 6891. Springer (2011)

6. Ghosh, A., Papadopoulo, T., Deriche, R.: Biomarkers for HARDI: 2nd \& 4th order tensor invariants. ISBI, Barcelona (May 2012)

7. Ghosh, A., Papadopoulo, T., Deriche, R.: Generalized invariants of a 4th order tensor: Building blocks for new biomarkers in dMRI. In: Panagiotaki, E., ODonnell, L., Schultz, T., Zhang, G.H. (eds.) CDMRI Workshop, MICCAI, pp. 165-173 (2012)

8. Schwab, E., Cetingül, H.E., Afsari, B., Yassa, M.A., Vidal, R.: Rotation invariant features for HARDI. In: Gee, J.C., Joshi, S., Pohl, K.M., Wells, W.M., Zöllei, L. (eds.) IPMI 2013. LNCS, vol. 7917, pp. 705-717. Springer, Heidelberg (2013)

9. Barmpoutis, A., Hwang, M.S., Howland, D., Forder, J.R., Vemuri, B.C.: Regularized positive-definite fourth-order tensor field estimation from DW-MRI. NeuroImage 45(1), S153-S162 (2009)

10. Ghosh, A., Moakher, M., Deriche, R.: Ternary quartic approach for positive 4 th order diffusion tensors revisited. ISBI: From Nano to Macro, pp. 618-621 (June 2009)

11. Barmpoutis, A., Zhuo, J.: Diffusion kurtosis imaging: Robust estimation from DWMRI using homogeneous polynomials. In: ISBI 2011, pp. 262-265 (2011)

12. Anwander, A., Tittgemeyer, M., von Cramon, D.Y., Friederici, A.D., Knosche, T.R.: Connectivity-Based Parcellation of Broca's Area. Cerebral Cortex 17(4), 816$825(2007)$ 